12

\title{
Использование полос стабильности для улучшения характеристик квадрупольных фильтров масс
}

\author{
(C) М.Ю. Судаков, Е.В. Мамонтов
}

Рязанский государственный радиотехнический университет, 390035 Рязань, Россия

e-mail: m.soudakov@yandex.ru

(Поступило в Редакцию 10 марта 2016 г. В окончательной редакции 23 июня 2016 г.)

Обсуждена возможность повышения разрешающей способности квадрупольных фильтров масс. Показано, что ограничения, связанные с конечным временем пролета, накладываемые „правилом Цана“, видоизменяются при использовании „островков стабильности“, возникающих при возбуждении квадруполя дополнительными сигналами. Путем расчета экспоненциального инкремента нарастания амплитуды колебаний объяснен эффект ускорения сортировки и улучшения формы массовых пиков при фильтрации ионов в островках стабильности. Теоретически исследован случай возбуждения двумя сигналами на разных частотах. Рассчитаны условия, при которых происходит подавление резонанса в первом порядке для одного из направлений движения. Прямое моделирование формы массового пика фильтра масс показывает возможность получения разрешающей способности 10000 при времени пролета ионов через квадруполь в 100 циклов основного питания, а также нечувствительность нового режима работы к нелинейным искажениям в квадруполях со стержнями круглого сечения.

DOI: 10.21883/JTF.2017.01.44025.1797

\section{Введение. Проблемы повышения разрешающей способности квадрупольных фильтров масс}

Разрешение масс селекции, по определению, равно ширине интервала масс $\Delta M=M_{\max }-M_{\min }$, пропускаемых фильтром на выход из квадруполя и попадающих на детектор или в следующую стадию масс анализа. Разрешающая способность $R$ является безразмерной величиной, равной отношению номинальной массы $M_{\text {nom }}=\left(M_{\min }+M_{\max }\right) / 2$ к пропускаемому диапазону:

$$
R=\frac{M_{\mathrm{nom}}}{\Delta M} .
$$

Очевидно, что для фильтра масс, работающего в обычном режиме в вершине первой зоны стабильности (рис. 1 из [1]), границы массового диапазона можно связать с параметрами $q$, соответствующими границам стабильной зоны, и получить выражение для теоретической разрешающей способности

$$
R_{\text {theor }}=\frac{q_{1}}{q_{\max }-q_{\min }},
$$

где $q_{1}=0.705996$ - значение, соответствующее вершине первой зоны. На практике разрешающую способность, согласно формуле (1), определяют по форме пика для ионов одинаковой массы, измеряя ширину пика на каком-то уровне (обычно 10\%) от максимума.

Еще на заре развития квадрупольной масс-спектрометрии было обнаружено [2], что теоретическая разрешающая способность (2) достигается только, если ионы проводят достаточное время внутри квадруполя. В противном случае фактическая разрешающая способность пропорциональна квадрату времени пролета $n$ ионов через квадруполь, измеренному в периодах высокочастотного питания (RF или ВЧ):

$$
R=\frac{n^{2}}{C_{Z}} .
$$

Здесь $C_{Z}-$ константа, величина которой зависит от ожидаемого теоретического разрешения и уровня, на котором измеряется ширина пика $\left(C_{Z}=10-20\right.$ для $10 \%$ от максимума в вершине первой зоны [3,4]). Этот закон, устанавливающий фундаментальное ограничение на разрешающую способность фильтров масс, работающих в обычном режиме, в дальнейшем будем называть „правилом Цана“. Согласно этому правилу, при времени пролета в 100 циклов, например, максимальная разрешающая способность равна 500.

Для улучшения разрешающей способности требуется увеличить время пролета ионов через квадруполь. Для этого имеется несколько возможностей. Во-первых, можно изготавливать длинные квадруполи. История масс-спектрометрии знает пример, когда был создан квадруполь длиной $5.82 \mathrm{~m}$ (стержни располагались вертикально, чтобы избежать изгибов), на котором была получена разрешающая способность 16000 [5]. М.Н. Amad и R.S. Houk [6] использовали многократные отражения, чтобы реализовать многопроходной режим движения ионов в квадруполе и продемонстрировали разрешающую способность 22000 . На практике квадруполи с радиусом поля в 4-6 mm изготавливаются с длиной стержней 100-300 mm, так как требуемая точность электродов и сборки лежит в микронном диапазоне. Во-вторых, для увеличения времени пролета ионов можно уменьшать продольную энергию пучка (скорость 
ионов). Для оценок возьмем квадруполь с типичными параметрами - радиусом поля $5 \mathrm{~mm}$ при длине стержней $200 \mathrm{~mm}$, работающий на частоте $1 \mathrm{MHz}$. В такой системе однозарядные ионы массой $609 \mathrm{Da}$ при продольной энергии $12 \mathrm{eV}$ пролетают квадруполь за 100 циклов RF. Максимальная разрешающая способность, согласно правилу Цана, в этом случае составит 500. Многие из источников ионов обладают собственным разбросом энергии в несколько электронвольт, поэтому затруднительно уменьшить энергию пучка менее $10 \mathrm{eV}$. B-третьих, можно использовать RF-питание более высокой частоты. Такой подход ограничен максимальным напряжением, которое можно подать на стержни, не опасаясь пробоя, так как увеличение частоты, например, в 2 раза требует в 4 раза более высокого напряжения, чтобы привести ионы той же массы в вершину первой зоны стабильности вблизи $q_{1}=0.705996$. Даже если эти проблемы преодолены, увеличение времени пролета ионов внутри квадруполя в такой же пропорции увеличивает время пролета областей переходного поля. В этих областях поле значительно отличается от квадрупольного и движение ионов является нестабильным, что приводит к пространственному и энергетическому рассеяниям пучка и драматическому падению трансмиссии.

Вышеприведенное обсуждение показывает, что возможности увеличения разрешающей способности квадрупольных фильтров масс, работающих в обычном режиме, весьма ограничены и в настоящее время практически исчерпаны. Наилучшим результатом на данный день является разрешающая способность около 5000 (при разумной трансмиссии) и достигается это в высокоточных квадруполях с гиперболическим профилем стержней, с использованием столкновительного охлаждения пучка, префильтров, а также RF-питания с повышенной частотой и большим напряжением.

Есть и другой путь преодоления ограничений, накладываемых правилом Цана, а именно изменение этого правила. Помимо первой зоны стабильности имеется бесконечное число высших общих зон стабильности, лежащих при больших значениях параметров $a$ и $q$. Н.В. Коненков и др. [7] использовали фильтрацию в III промежуточной зоне стабильности для создания анализатора состава остаточных газов с разрешающей способностью 5000. Фильтрация ионов в других высших зонах стабильности детально исследовалась в лаборатории Д. Дугласа [8-10]. В этих зонах сортировка ионов происходит намного быстрее и правило Цана видоизменяется. В частности, для II зоны В.В. Титов [11] и другие исследователи [8] дают зависимость максимальной разрешающей способности от времени пролета $R_{1 / 2}=24 n^{2}$, что соответствует $C_{Z} \approx 0.04$. Использование высших зон стабильности дает очевидный способ улучшения разрешающей способности, однако это сопровождается массивным падением трансмиссии, делающим такой способ неприемлемым. Проблема в том, что высшие зоны лежат при очень больших значениях параметров $a$ и $q$, которые достигаются только внутри квадруполя, а в переходных областях движение ионов сильно нестабильно, что приводит к разбросу поперечной энергии пучка.

В работе [1] было показано, что островки стабильности, создаваемые квадрупольным возбуждением, являются, по существу, аналогом высших зон стабильности. Однако они расположены на том же расстоянии от центра диаграммы стабильности, что и вершина первой зоны, а кроме того, представляют намного больше гибкости в управлении скоростью сортировки ионов, чем обычные высшие зоны. В настоящей работе будут исследованы свойства островков стабильности и вскрыта причина ускорения сортировки и улучшения формы пиков. Будет показано, как можно сформировать островки в полосы стабильности, наиболее удобные для фильтрации. В заключение свойства нового режима работы фильтров масс исследуются методами прямого моделирования, наглядно иллюстрируя его преимущества.

\section{Ускорение сортировки ионов при использовании квадрупольного возбуждения}

Улучшение формы линии при использовании квадрупольного возбуждения, видимо, впервые было замечено К. Мисеки (см. исторический обзор в [12]). Патент U.S. No. 5.227.629 описывает квадрупольный фильтр масс, в котором помимо обычного высокочастотного RF и постоянного разрешающего напряжения DC приложено дополнительное переменное (AC) напряжение $V_{\mathrm{ex}} \cdot \cos \left(\omega_{\mathrm{ex}} t\right)$. Сигнал возбуждения имеет частоту $\omega_{\mathrm{ex}}$, отличную от частоты основного питания RF, и создает полосы нестабильности вблизи вершины первой зоны стабильности, что приводит к эффективному устранению хвостов массовых пиков, тем самым улучшая разрешение масс сепарации и надежность результатов измерений. В работе [13] было показано, что использование островков стабильности для сортировки позволяет улучшить чувствительность к разделению соседних масс (изотопическую чувствительность) на 2-3 порядка. Очевидно, что данные эффекты свидетельствуют о более эффективной сортировке ионов в островках стабильности.

Сокращение необходимого времени сортировки объясняется возрастанием уровня нестабильности вблизи островков стабильности. Последний можно характеризовать инкрементом нарастания колебаний $\mu$, который вычисляется через след матрицы монодромии (см. уравнение (12) в [1]) для нестабильных решений:

$$
\cos h(\mu)=0.5 \cdot\left|m_{11}+m_{22}\right| .
$$

Величина $\exp (\mu)$ показывает, во сколько раз возрастает амплитуда колебаний за один период ВЧ. Расчет матрицы монодромии можно выполнить методом, описанным в [1]. Рис. 1 показывает расчет скорости нарастания амплитуды колебаний вдоль рабочей прямой в двух случаях: при нормальной работе квадруполя 
$a$

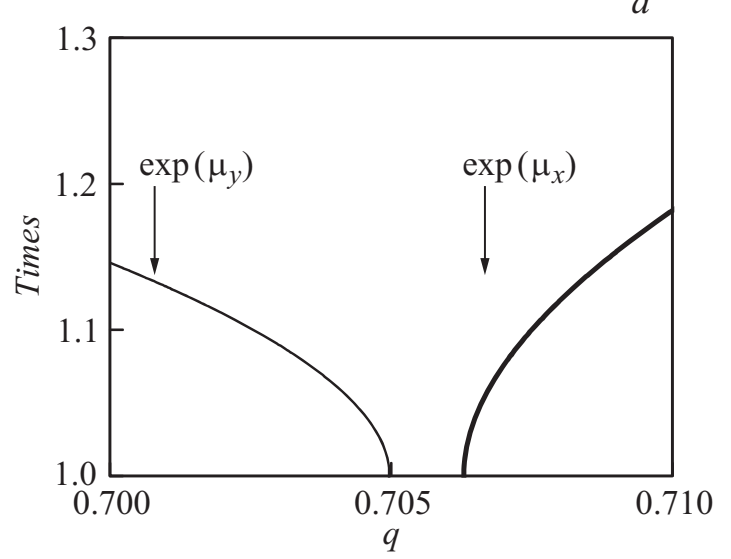

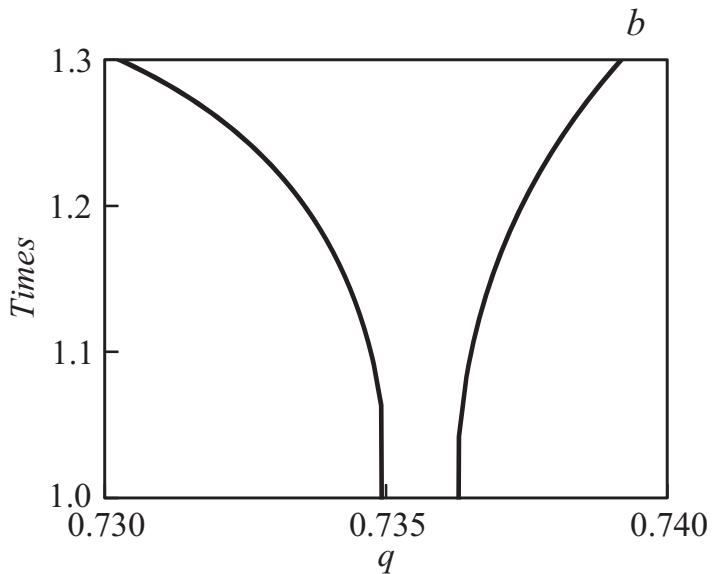

Рис. 1. Скорость нарастания амплитуды колебаний в нестабильной области вдоль линии сканирования. $a-$ для нормальной работы квадруполя вблизи вершины первой зоны стабильности, наклон рабочей прямой $\lambda=0.1676$. $b-$ для квадруполя с возбуждением на частоте $v=9 / 10, q_{e x}=0.006, \lambda=0.161$ при сортировке вблизи островка, находящегося с правой стороны диаграммы стабильности (островок „С“). В обоих случаях квадруполь настроен на теоретическую разрешающую способность около 500. Жирная линия - нестабильность колебаний вдоль $X$, тонкая - вдоль $Y$.

и при использовании квадрупольного возбуждения. Из рис. 1, $a$ видно, что при нормальной работе квадруполя сортировка со стороны больших значений параметра $q$ (граница пика со стороны легких ионов) происходит за счет нестабильности движения вдоль $X$, а со стороны меньших значений $q$ (тяжелые ионы) - за счет нестабильности $Y$-движения. Невысокая скорость сортировки на $Y$-границе приводит к появлению значительных хвостов пика и представляет проблему для эффективной фильтрации в обычном режиме.

В противоположность этому для квадруполя с возбуждением при использовании сортировки в островке ста-

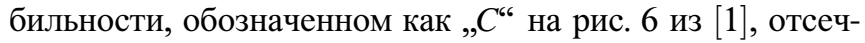
ка нежелательных масс с обеих сторон полосы пропускания обеспечивается за счет нестабильности $X$-движения (см. рис. $1, b)$. Скорость нарастания колебаний вблизи островка „, $C^{6}$ значительно больше (если учесть экспоненциальное нарастание амплитуды в течение 100 периодов), чем в нормальном режиме, изображенном на рис. 1,a. Эффект возрастания инкремента нарастания колебаний объясняется развитой в предыдущей работе теорией. Действительно, согласно уравнению огибающей (26) и соответствующей диаграмме (рис. 4 из [1]), сортировка в островке „С“ происходит в узкой полосе первой зоны стабильности. Так, для параметра $Q_{x}$ при условиях рис. 1 расчет дает значение 3.002. Из рис. 4 в [1] можно видеть, что в этом случае соседствующие со стабильной зоной точки будут обладать высоким инкрементом нестабильности.

В работе [14] отмечалось, что островок „,C“ наиболее эффективен для сортировки, во-первых, из-за одномерного характера фильтрации (только вдоль направления $X$ ) и, во-вторых, потому, что рабочая прямая пересекает его почти под прямым углом. К сожалению, при использовании простого квадрупольного возбуждения рабочая прямая на пути к островку „С“ пересекает и другие стабильные области, что приводит к наложению спектров. Расчет показывает, что при использовании простого квадрупольного возбуждения эту проблему нельзя преодолеть, но это возможно при возбуждении двумя сигналами на разных частотах. Анализ этого случая с помощью теории, развитой в предыдущей работе [1], является предметом следующего раздела.

\section{Теоретическое исследование случая возбуждения двумя сигналами}

При квадрупольном возбуждении двумя гармоническими сигналами на разных частотах результирующая функция питания $V(t)$ равна

$$
\begin{aligned}
V(t)=U & +V \cos \Omega t+V_{\mathrm{ex} 1} \cos \left(\omega_{\mathrm{ex} 1} t+\alpha_{1}\right) \\
& +V_{\mathrm{ex} 2} \cos \left(\omega_{\mathrm{ex} 2} t+\alpha_{2}\right) .
\end{aligned}
$$

Здесь $\omega_{\text {ex1 }}$ и $\omega_{\text {ex2 }}$ - циклические частоты двух возбуждений (будем считать, что первая частота меньше: $\left.\omega_{\mathrm{ex} 1}<\omega_{\mathrm{ex} 2}\right), V_{\mathrm{ex} 1}$ и $V_{\mathrm{ex} 2}-$ амплитуды напряжения первого и второго возбуждений, $\alpha_{1}$ и $\alpha_{2}$ - начальные фазы возбуждений по отношению к основному RF. Введем безразмерные параметры согласно уравнениям

$$
\begin{gathered}
v_{1}=\frac{\omega_{\mathrm{ex} 1}}{\Omega}, \quad v_{2}=\frac{\omega_{\mathrm{ex} 2}}{\Omega}, \\
q_{\mathrm{ex} 1}=\frac{4 e V_{\mathrm{ex} 1}}{M \Omega^{2} r_{0}^{2}}, \quad q_{\mathrm{ex} 2}=\frac{4 e V_{\mathrm{ex} 2}}{M \Omega^{2} r_{0}^{2}} .
\end{gathered}
$$

В первом порядке теории возмущений, развитой в работе [1], данные два возбуждения можно рассматривать по отдельности и суммировать их воздействие. Для каждого из них поправки к следу матрицы монодромии имеют 
вид, как в уравнении (21) из [1] при величинах $c_{11}$ и $s_{11}$, вычисленных для соответствующей частоты. Ограничимся случаем, когда относительные частоты возбуждений равны

$$
v_{1}=v, \quad v_{2}=1-v .
$$

Здесь $v-$ „базовая“ частота, фактически равная частоте первого возбуждения, а частота второго возбуждения отстроена от основного RF на ту же величину в меньшую сторону. Для упрощения будем также считать, что начальные фазы обоих возбуждений нулевые: $\alpha_{1}=\alpha_{2}=0$. Суммируя поправки к следу матрицы монодромии от обоих сигналов, получим

$$
\begin{aligned}
& s(n)=s_{0}-q_{\mathrm{ex} 1} \Pi_{x} \cdot\left\lfloor c_{11, x}(v) \cos (2 v n \pi)\right. \\
& \left.-s_{11, x}(v) \sin (2 v n \pi)\right\rfloor-q_{\mathrm{ex} 2} \Pi_{x} \cdot\left[c_{11, x}(1-v)\right. \\
& \left.\times \cos (2(1-v) n \pi)-s_{11, x}(1-v) \sin (2(1-v) n \pi)\right] .
\end{aligned}
$$

В последнем слагаемом в тригонометрических функциях можно исключить величины, равные полному периоду $2 \pi n$, и тогда, приведя подобные слагаемые, получим следующее выражение

$$
\begin{aligned}
& s(n)=s_{0}-\Pi_{x} \cdot\left\lfloor q_{\mathrm{ex} 1} c_{11, x}(v)-q_{\mathrm{ex} 2} c_{11, x}(1-v)\right\rfloor \cos (2 v n \pi) \\
& \quad+\Pi_{x} \cdot\left[q_{\mathrm{ex} 1} s_{11, x}(v)-q_{\mathrm{ex} 2} s_{11, x}(1-v)\right] \cdot \sin (2 v n \pi) .
\end{aligned}
$$

Повторяя рассуждения, приведшие к уравнению (26) в работе [1], получим, что и в случае двух возбуждений с указанными параметрами огибающая колебаний подчиняется уравнению Матье с параметрическим возбуждением, характеризуемым величиной

$Q_{x}(v)=\frac{\Pi_{x}}{\pi^{2} v^{2}} \sqrt{\begin{array}{c}{\left[q_{\mathrm{ex} 1} c_{11, x}(v)-q_{\mathrm{ex} 2} c_{11, x}(1-v)\right]^{2}+} \\ +\left[q_{\mathrm{ex} 1} s_{11, x}(v)-q_{\mathrm{ex} 2} s_{11, x}(1-v)\right]^{2}\end{array}}$.

Для огибающей колебаний иона вдоль направления $Y$ справедливо уравнение Матье (29) в [1], в котором интенсивность параметрического возбуждения характеризуется величиной $Q_{y}(v)$, по виду совпадающей с выражением (10). Целесообразно исследовать поведение величин $Q_{x}(v)$ и $Q_{y}(v)$ от параметров возбуждения. Для примера возьмем значение частоты $v=0.05$ и зафиксируем амплитуду второго возбуждения $q_{\mathrm{ex} 2}=0.02$. Расчет зависимости величин $Q_{x}(v)$ и $Q_{y}(v)$ от отношения амплитуд возбуждений $b=q_{\mathrm{ex} 1} / q_{\mathrm{ex} 2}$ представлен на рис. 2. Удивительно, что сложное выражение (10) на поверку оказывается простой линейной функцией. Учитывая этот факт, раскроем подкоренное выражение в уравнении (10) как

$$
\begin{aligned}
& q_{\mathrm{ex} 2}^{2} c_{11, x}(v)^{2}+2 q_{\mathrm{ex} 1} q_{\mathrm{ex} 2} c_{11, x}(v) c_{11, x}(1-v) \\
& +q_{\mathrm{ex} 2}^{2} c_{11, x}(1-v)^{2}+q_{\mathrm{ex} 1}^{2} s_{11, x}(v)^{2} \\
& -2 q_{\mathrm{ex} 1} q_{\mathrm{ex} 2} s_{11, x}(v) s_{11, x}(1-v)+q_{\mathrm{ex} 2}^{2} s_{11, x}(1-v)^{2}
\end{aligned}
$$

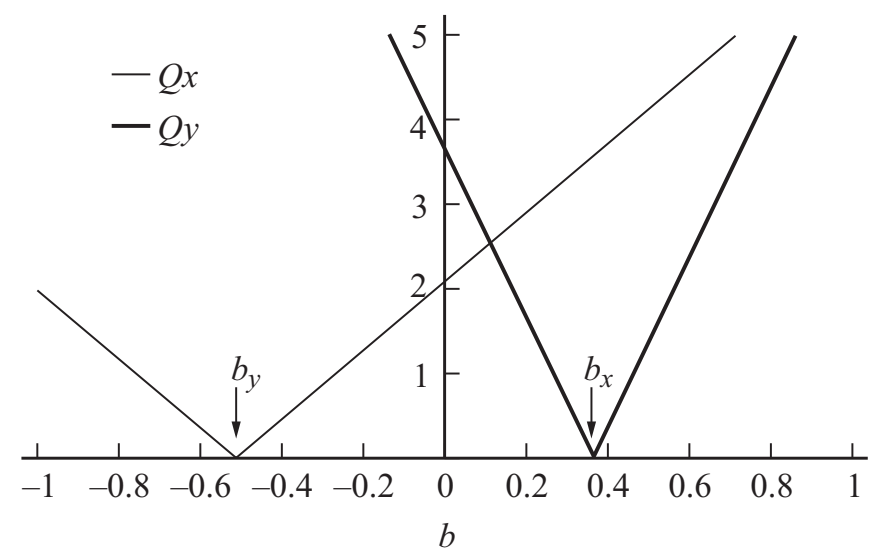

Рис. 2. Зависимость амплитуд параметрического возбуждения в уравнениях огибающих колебаний ионов в зависимости от отношения $b=q_{\mathrm{ex} 1} / q_{\mathrm{ex} 2}$ при фиксированных значениях $v=0.05$ и $q_{\mathrm{ex} 2}=0.02$.

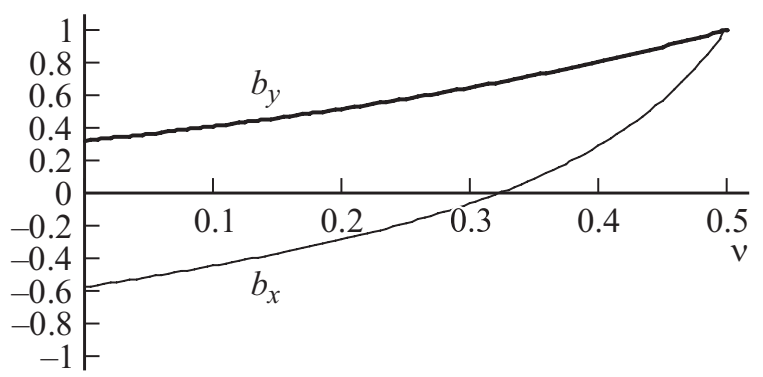

Рис. 3. Зависимость критического отношения амплитуд возбуждений от базовой частоты.

и представим уравнение (10) в виде линейной функции отношения амплитуд возбуждения $q_{\mathrm{ex} 1} / q_{\mathrm{ex} 2}$ :

$Q_{x}(v)=\frac{\Pi_{x}}{\pi^{2} v^{2}} q_{\mathrm{ex} 2} \sqrt{c_{11, x}(v)^{2}+s_{11, x}(v)^{2}} \cdot\left|\frac{q_{\mathrm{ex} 1}}{q_{\mathrm{ex} 2}}-b_{x}(v)\right|$,

где

$b_{x}(v)=-2 \frac{c_{11, x}(v) \cdot c_{11, x}(1-v)-s_{11, x}(v) \cdot s_{11, x}(1-v)}{c_{11, x}^{2}(v)+s_{11, x}^{2}(v)}$.

В случае, когда отношение амплитуд возбуждения равно $b_{x}$, величина $Q_{x}$ обращается в нуль, что означает, что движение по $X$ не подвергается параметрическому возбуждению дополнительными сигналами (в первом порядке). Фактически они компенсируют влияние друг друга. Эти значения отношения амплитуд естественно назвать „критическими“. На рис. 3 рассчитана зависимость критических отношений от частоты низкочастотного возбуждения в диапазоне от 0 до 0.5. Заметим, что развиваемая теория справедлива для достаточно малых значений частоты, поэтому только начальная часть графиков 10 имеет значение. В этой области критические отношения линейно изменяются с частотой. В частности, для $v=0.05$ имеем $b_{x}=-0.514$ 

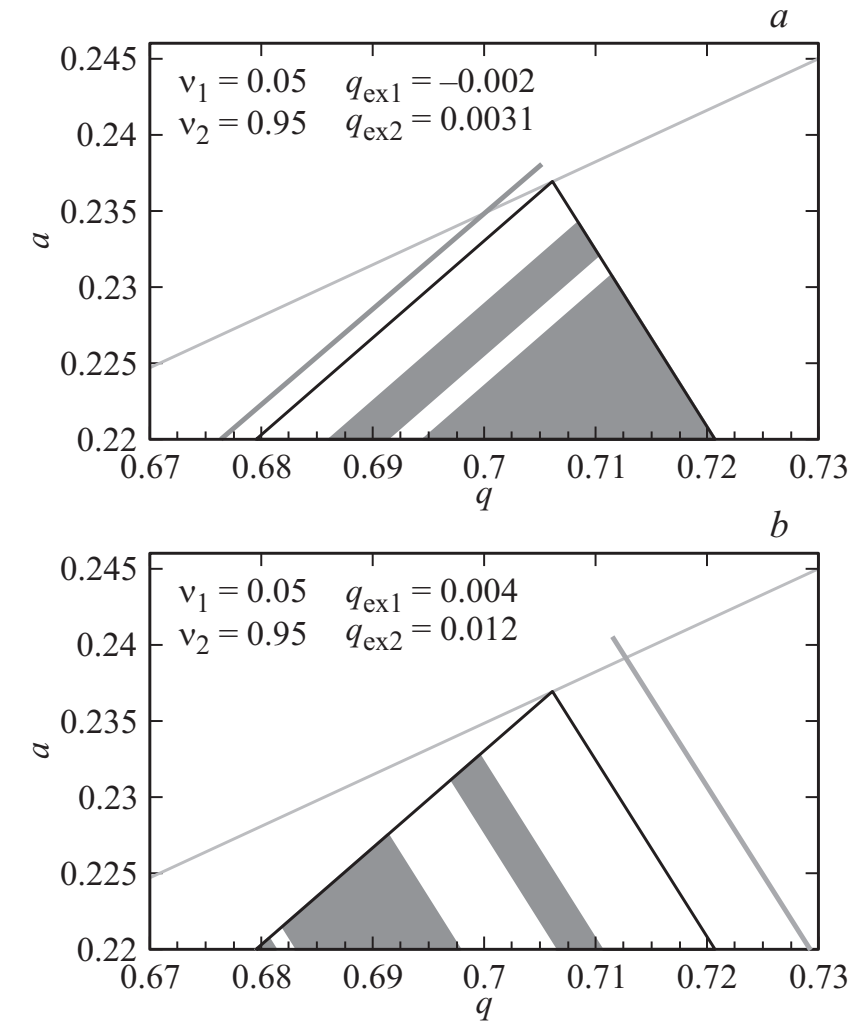

Рис. 4. Расчет диаграммы стабильности вблизи вершины первой зоны при наличии двух возбуждающих сигналов с параметрами, указанными в легендах графиков. Границы исходной стабильной области показаны жирной линией. Тонкая линия рабочая прямая, проходящая через вершину исходного треугольника стабильности.

и $b_{y}=0.363$. Когда отношение амплитуд возбуждений равно $b_{x}$, в диаграмме стабильности отсутствует расщепление границы с правой стороны (для движения вдоль $X$ ). Это иллюстрируется точным расчетом диаграммы на рис. $4, a$. В результате отсутствия расщепления вместо островков образуется длинная полоса стабильности с левой стороны. В другом случае, когда отношение амплитуд равно критическому значению $b_{y}$, отсутствует расщепление левой границы стабильности. В этом случае образуется длинная полоса стабильности с правой стороны (рис. 4, $b$ ), которую в дальнейшем будем называть „X-band“. Заметим, что оптимальные критические значения, найденные из прямого расчета диаграмм на рис. 4 , незначительно отличаются от теоретических, полученных из уравнения (13).

На рис. 4 тонкой линией показано положение „рабочей прямой“", проходящей через вершину исходной первой зоны стабильности $(U / V=0.1678)$. Ионы различных масс имеют значения параметров вдоль этой прямой. Только узкий диапазон масс попадает в полосу стабильности. Разрешающая способность определяется шириной этой полосы, которая в свою очередь задается амплитудами дополнительных возбуждений. Сканирова- ние спектра с использованием полосы стабильности осуществляется как обычно, путем изменения амплитуды основного RF и постоянного смещения DC, сохраняя отношение $U / V$ постоянным. Для сохранения положения и формы полосы стабильности необходимо также пропорционально увеличивать амплитуды АС-возбуждений.

Очевидно, случай, изображенный на рис. $4, b$, наиболее удобен для фильтрации из-за относительного расположения полосы стабильности и рабочей прямой, которые пересекаются почти под прямым углом. Это означает, что малые флуктуации питающих напряжений меньше влияют на стабильность ионов. Кроме этого, нестабильность траекторий, а следовательно, и скорость сортировки нарастают значительно быстрее при удалении от стабильной полосы вдоль рабочей прямой. В заключение заметим, что результата, подобного изображенному на рис. $4, b$, можно добиться и при других значениях частот возбуждающих сигналов, например $v_{1}=v$ и $v_{2}=1+v$ или $v_{1}=1-v$ и $v_{2}=2-v$ и т.д. Критические отношения амплитуд в этих случаях будут другими и могут быть вычислены.

\section{Моделирование формы массового пика}

Численное моделирование движения ионов в квадрупольном фильтре масс - наиболее прямой способ проверки нового режима работы и сравнения его свойств с обычным режимом. Для моделирований был выбран квадруполь с типичными параметрами, радиусом поля $r_{0}=5 \mathrm{~mm}$ при длине стержней $200 \mathrm{~mm}$, запитанный на RF-частоте $1 \mathrm{MHz}$. Электрическое поле считается идеальным квадрупольным по всей длине, таким образом, переходные поля не учитываются на данном этапе. В нормальном режиме работы квадруполь настроен на селекцию однозарядных ионов массой $609 \mathrm{Da}$ с теоретической разрешающей способностью 10000 . Соответствующие параметры питания приведены в таблице. Для моделирования нового режима работы с использованием $\mathrm{X}$-band применялись возбуждения на относительных частотах $v_{1}=v$ и $v_{2}=1-v$ при $v=0.05$. Параметры возбуждений настроены на теоретическую разрешающую способность 10000 (см. таблицу).

Пучок ионов одинаковой массы подается на вход квадруполя с равной продольной энергией, чтобы обеспечить одинаковое время пролета ионов. Пучок имеет

Параметры питающих напряжений для моделирований работы фильтра масс в обычном режиме (третья строка) и в режиме с использованием X-band (четвертая строка). Оба режима настроены на теоретическую разрешающую способность 10000. Напряжения даны как zero-peak, pole-to-ground

\begin{tabular}{l|c|c|c|c}
\hline Параметр & $\mathrm{DC}$ & $\mathrm{RF}$ & $\mathrm{AC}-1$ & $\mathrm{AC}-2$ \\
\hline Частота, KHz & 0 & 1000 & 50 & 950 \\
Обычный режим & $184.531 \mathrm{~V}$ & $1099.507 \mathrm{~V}$ & 0 & 0 \\
X-band-I & $184.554 \mathrm{~V}$ & $1099.507 \mathrm{~V}$ & $10.590 \mathrm{~V}$ & $31.135 \mathrm{~V}$
\end{tabular}


нормальный разброс поперечных положений ионов со стандартным отклонением $0.1 \mathrm{~mm}$ и нормальный разброс поперечных скоростей, соответствующий среднеквадратичной энергии $0.025 \mathrm{eV}$. Время появления ионов имеет равномерный разброс в пределах от 0 до $20 \mu \mathrm{s}$ (в течение 20 периодов RF), для того чтобы равномерно покрыть все возможные фазы АC-возбуждений. В каждом цикле моделирования 10000 ионов трассируются до тех пор, пока они не столкнутся с электродами или не пройдут на выход квадруполя. Прошедшие ионы подсчитываются, и моделирование повторяется для ионов другой массы, давая, таким образом, форму массового пика квадруполя. Для расчета траекторий использовался метод Рунге-Кутты 4-го порядка [14] с постоянным шагом $7.8125 \mathrm{~ns}$ (128 шагов на один период RF). Из расчетов диаграмм известно, что даже 32 шага на один период достаточно для адекватного описания стабильности ионов.

\section{Сравнение с обычным режимом работы}

Результаты моделирований приведены на рис. 5. В случае обычного режима работы пики имеют значительные „хвосты“, и фактическая разрешающая способность ограничена временем пролета ионов. Энергия пучка $12 \mathrm{eV}$ соответствует времени пролета в 100 периодов RF и приводит к разрешающей способности 575 (на уровне
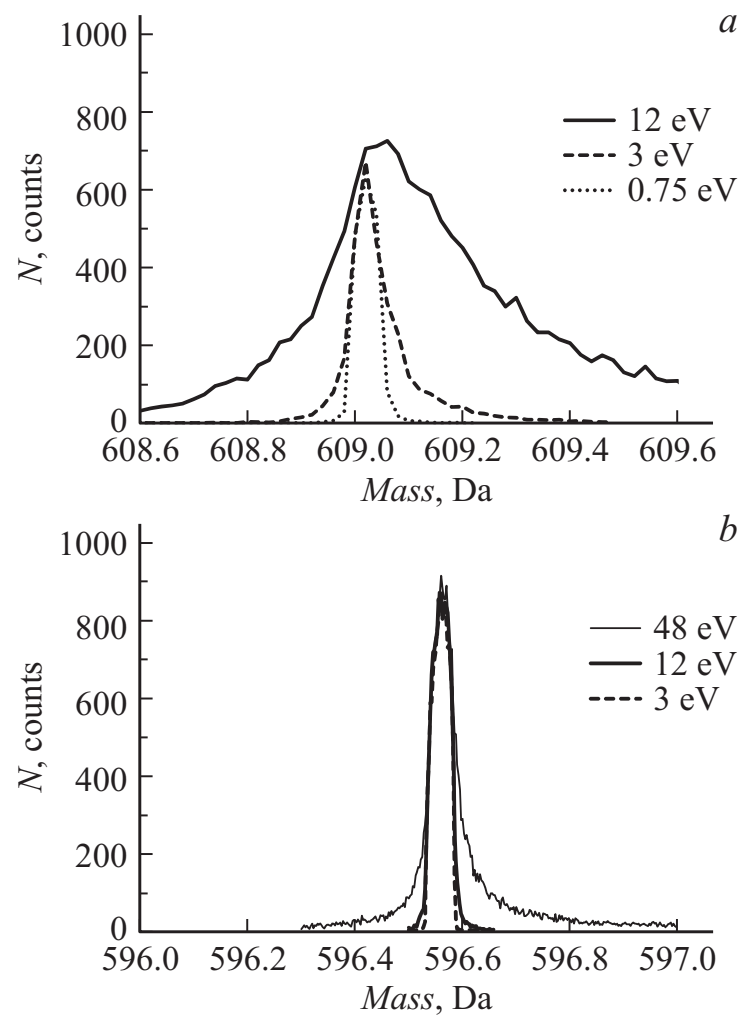

Рис. 5. Результаты моделирований формы пиков в квадруполе со стержнями гиперболического профиля в обычном режиме $(a)$ и с использованием X-band $(b)$ при различной продольной энергии пучка.

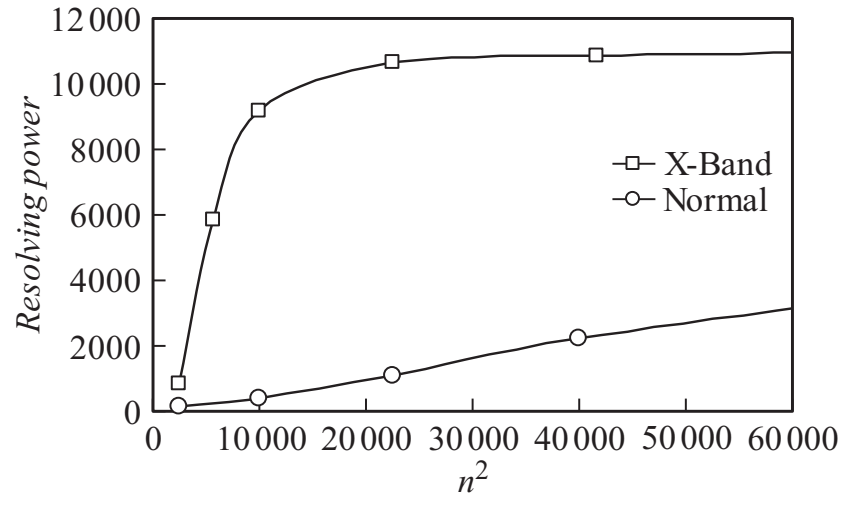

Рис. 6. Зависимость фактической разрешающей способности от квадрата числа периодов RF, проводимых ионом в поле фильтра для нормального режима и с использованием X-band. Оба режима настроены на теоретическую разрешающую способность 10000 .

$10 \%$ от максимума пика). Меньшая энергия $3 \mathrm{eV}$ (200 периодов) дает $R=2175$, и энергия $0.75 \mathrm{eV}$ (400 периодов) дает $R=4350$. Максимум пика соответствует 700 ионам из 10000 . Из-за разброса начальных поперечных положения и скоростей 93\% ионов оседают на электродах, хотя их траектории номинально стабильны. В новом способе фильтрации с использованием X-band (рис. 5, внизу) хвосты пиков значительно меньше и при энергии пучка в $3 \mathrm{eV}$ практически отсутствуют. В результате даже при энергии пучка в $12 \mathrm{eV}$ разрешающая способность на уровне $10 \%$ от максимума равна 9178. Максимум пика соответствует 900 отсчетам, что на 28\% больше, чем в обычном режиме. Даже при энергии пучка в $48 \mathrm{eV}$ (50 периодов RF) разрешающая способность равна 4000, что в нормальном режиме получается только при энергии пучка в 64 раза меньшей. Заметим, что в новом режиме максимум пика приходится на массу 596.56 Da из-за сдвига X-band в сторону более высоких значений $q$, что и предсказывается расчетом диаграммы стабильности.

Эти результаты суммированы на рис. 6, где представлена разрешающая способность в зависимости от квадрата числа периодов RF, проводимых ионом в поле фильтра. Для нормального режима зависимость следует правилу Цана с $C_{Z} \approx 20$. В результате даже время пролета в 200 периодов приводит к $R=2000$, тогда как при использовании X-band разрешающая способность в 10000 достигается практически при времени пролета в 100 периодов.

\section{Нечувствительность к нелинейным искажениям поля}

Изготовление и сборка квадруполей со стержнями гиперболического профиля является трудной и дорогостоящей задачей. Большинство коммерческих квадруполей фактически изготавливается из стержней круглого 

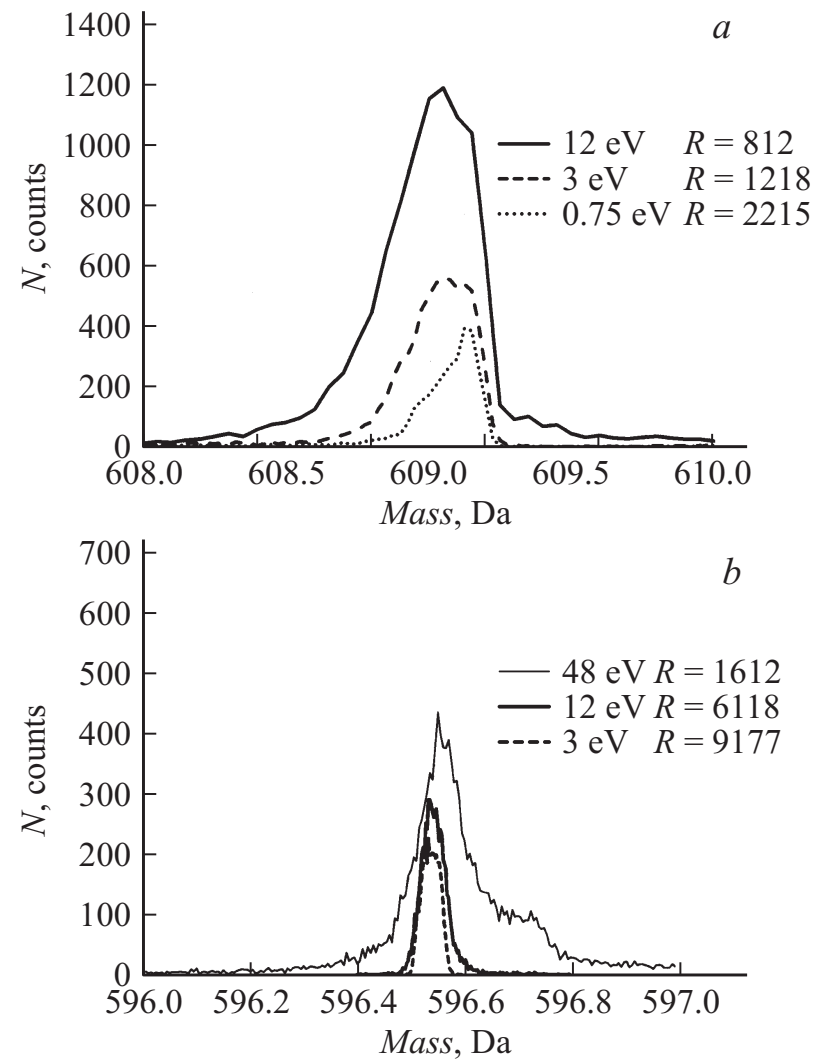

Рис. 7. Форма пика в квадруполе со стержнями круглого сечения $\left(R / r_{0}=1.120\right)$ в нормальном режиме $(a)$ и с использованием X-band $(b)$ при различной энергии пучка. В нормальном режиме квадруполь настроен на теоретическую разрешающую способность -2000 (выше вершины первой зоны). Для режима $\mathrm{X}$-band квадруполь настроен на теоретическую разрешающую способность 10000 . Фактическая разрешающая способность приведена в легендах графиков. Обе шкалы на графике $b$ имеют вдвое меньший масштаб, чем на графике $a$.

сечения (цилиндров). Электрическое поле внутри таких квадруполей отличается от идеального и содержит нелинейные искажения. Из экспериментальной практики и теоретических исследований было определено оптимальное отношение [15] радиуса стержней $R$ к радиусу поля $r_{0}$, которое лежит в диапазоне от 1.120 до 1.130 . В такой геометрии электродов положительные и отрицательные искажения частично компенсируют друг друга и дают поле, наиболее близкое к квадрупольному. К сожалению, эта компенсация никогда не бывает полной, и квадруполи с цилиндрическими стержнями обычно работают хуже гиперболических в смысле разрешающей способности и трансмиссии.

Для моделирований поле было вычислено способом, описанным в [16]. Это модификация метода граничных элементов, позволяющая рассчитать потенциал электрического поля в квадруполе со стержнями круглого сечения с точностью 10 знаков. Потенциалы поля были сохранены в формате файлов программы SIMION [17] на равномерной сетке с шагом $25 \mu \mathrm{m}$ и использовались в моделировании для расчета узловых значений электрических полей с последующей билинейной интерполяцией для точек между узлами. Результаты расчета формы пиков для квадруполя с $R / r_{0}=1.120$ представлены на рис. 7. В нормальном режиме квадруполь настроен на селекцию однозарядных ионов массой $609 \mathrm{Da}$ с теоретической разрешающей способностью -2000. То есть рабочая прямая проходит выше вершины первой зоны стабильности. Именно при таком положении рабочей прямой получаются наилучшие результаты в смысле разрешающей способности. Из этого следует вывод, что квадруполи с цилиндрическими стержнями осуществляют сортировку ионов в нелинейном режиме, так как при отсутствии нелинейных искажений квадрупольного поля при таком положении рабочей прямой никакие ионы не проходят через квадруполь. Рис. 7, $a$ показывает, что наилучшая разрешающая способность достигается при энергии пучка $0.75 \mathrm{eV}$ и составляет 2215. Это вызвано значительными „хвостами“ и искажениями формы линии, вызванными нелинейными искажениями, присущими всем квадруполям с цилиндрическими стержнями во всем диапазоне $R / r_{0}=1.120-1.130$, считающемся оптимальным. В противоположность этому при использовании X-band для того же квадруполя пики не показывают нелинейных искажений, а хвосты полностью исчезают при энергии пучка $3 \mathrm{eV}$, приводя к разрешающей способности 9177. Максимум пика в этом случае примерно в 4 раза меньше, чем для квадруполя с гиперболическими стержнями (рис. $5, b$ ), что говорит о том, что нелинейные искажения поля все же сказываются деструктивно на трансмиссии, но не на разрешающей способности.

\section{Обсуждение результатов}

Препятствием на пути повышения разрешающей способности квадруполей, работающих в обычном режиме, является конечное время пролета ионов в квадрупольном поле и связанное с этим правило Цана. Видоизменение этого правила и ускорение сортировки возможно при использовании высших зон стабильности, прямым аналогом которых являются островки стабильности, возникающие из-за дополнительного возбуждения квадруполя на частоте, отличной от основного RF-питания. C помощью двух сигналов возбуждения удается подавить нестабильность движения в направлении $Y$ и сформировать островки стабильности в длинную узкую полосу (X-band) вблизи вершины первой зоны стабильности. Теоретическая разрешающая способность задается шириной полосы стабильности, которая в свою очередь задается амплитудой квадрупольного возбуждения. X-band наиболее удобна для масс селекции из-за взаимного расположения полосы стабильности и рабочей прямой, которые пересекаются почти ортогонально. Из-за этого стабильность ионов наименее подвержена влиянию малых флуктуаций питающих напряжений, а 
инкремент нестабильности траекторий вдоль рабочей прямой нарастает наиболее быстро при удалении от стабильной области. Это свойство иллюстрируется расчетом на рис. 1.

Заметим здесь, что форма и положение островков стабильности зависят от фазы АС-возбуждения относительно фазы RF, как следует из теории [1]. При реализации сигналов для формирования X-band следует обеспечить как точный фазовый синхронизм между двумя сигналами возбуждения $\mathrm{AC} 1$ и $\mathrm{AC} 2$, так и по отношению к основному ВЧ питанию, также точные значения частот $v_{1}=v$ и $v_{2}=1-v$. Представляется, что с современной цифровой техникой генерации сигналов это не составляет большой проблемы.

Второе важное преимущество - это одномерный характер сортировки, так как движение вдоль $Y$ остается стабильным вблизи X-band. Акцептансом прибора называется площадь области в фазовом пространстве начальных условий ионов, которые будут пропущены на выход прибора. Известно [18], что акцептанс фильтра масс вдоль каждого из поперечных направлений движения является малой величиной, пропорциональной $r_{0} f^{2}$, где $f$ - частота RF-питания. В обычном режиме работы в вершине первой зоны стабильности результирующий акцептанс является произведением двух малых величин - акцептансов вдоль $Y$ и вдоль $X$ и пропорционален $r_{0}^{2} f^{4}$. При фильтрации ионов в X-band движение вдоль $Y$ остается стабильным и акцептанс пропорционален $r_{0}^{2} f^{4}$. Это иллюстрируется расчетом формы пиков, приведенным на рис. 5, где максимум пиков в X-band на 28\% выше, чем в обычном режиме.

Рис. 6 показывает заметное возрастание скорости сортировки при использовании X-band по сравнению с обычным режимом. Для X-band начальный линейный участок кривой (при времени пролета менее 100 периодов RF) следует правилу Цана с $C_{Z} \approx 1$ и теоретическая разрешающая способность в 10000 достигается практически при времени пролета ионов в 100 циклов. Заметим в связи с этим, что при более высокой разрешающей способности с использованием X-band скорость сортировки будет еще выше, а соответствующая константа $C_{Z}$ меньше. Это следует из того, что более узкая зона X-band реализуется при более высоких уровнях возбуждения, а следовательно, более высоких значениях параметра $Q_{x}$ в уравнении огибающей. Соответственно инкремент нарастания колебаний вблизи стабильной полосы на рис. 4 в [1] будет более высоким. Это отличает островки стабильности от обычных высших зон, где величина константы $C_{Z}$ фиксирована и неуправляема.

Следует заметить, что одна только иллюстрация высокого разрешения, как описано выше, недостаточна для того, чтобы утверждать значимость нового режима работы для техники. Параметры $a$ и $q$, соответствующие стабильной зоне, достигаются только внутри квадруполя, а на этом пути ионы проходят область переходного поля, где параметры стабильности отличаются от номинальных, и могут соответствовать нестабильному движению, приводя к значительному рассеянию пучка. Именно это обстоятельство делает использование обычных высших зон стабильности непрактичным. В отличие от этого островки стабильности расположены в том же месте и на том же расстоянии от центра диаграммы стабильности, что и вершина первой зоны стабильности. Это позволяет использовать стандартные методы преодоления влияния переходных полей, такие как Delayed DC Ramp, или префильтр Брубакера [19]. Рамки данной публикации не позволяют привести результаты реалистичных моделирований квадруполей с интерфейсом столкновительного охлаждения с учетом переходных полей и Delayed DC Ramp. Можем только сказать здесь, что переходные поля не отменяют свойств и преимуществ X-band.

Замечательным свойством островков стабильности является их низкая чувствительность к нелинейным искажениям поля, иллюстрируемая расчетами на рис. 7. В работе [20] Д. Дуглас с соавторами показывает, что использование островков стабильности позволяет преодолеть значительные нелинейные искажения поля и получить разрешающую способность на уровне 2000 в квадруполях с сильно искаженной геометрией электродов. Это свойство также является следствием более высокой скорости сортировки, за несколько периодов AC-возбуждения (которое и осуществляет сортировку) нелинейные эффекты просто не успевают развиться.

\section{Выводы и заключение}

Квадрупольное возбуждение дополнительными сигналами является эффективным средством манипулирования диаграммой стабильности движения ионов в фильтре масс. Использование двух сигналов возбуждения позволяет сформировать диаграмму стабильности в виде полос, наиболее удобных для масс селекции. Свойства этих полос позволяют преодолеть многие ограничения, присущие обычному режиму работы:

- быстрая сортировка, когда всего несколько периодов базовой частоты АС-возбуждения $v$ достаточно для того, чтобы удалить нестабильные ионы, позволяет получать разрешающую способность на уровне 10000 при времени пролета ионов всего в 100 циклов основного RF-питания,

- сепарация ионов происходит в одном измерении, что увеличивает акцептанс устройства,

- области нестабильности, используемые для масс-сепарации, находятся только вблизи вершины первой зоны стабильности, что позволяет использовать технику delayed DC ramp для преодоления переходных полей и улучшения чувствительности,

- новый способ работы квадруполя оказывается нечувствителным ко многим видам искажений электродов.

Приведенные в работе примеры получены с использованием базовой частоты $v=0.05$, что является удобным практическим компромиссом - 100 периодов RF coдержит 5 периодов базового возбуждения. Аналогичные 
результаты могут быть получены с другими малыми значениями базовой частоты.

В режиме масс-анализа квадруполь собирает сигнал для каждой точки спектра в течение какого-то времени, постепенно сканируя RF- и DC-напряжения. Предполагая, что сигнал собирается как минимум в течение времени пролета ионов через квадруполь $(100 \mu \mathrm{s})$, сканирование диапазона 1 Da с разрешением 10000 требует как минимум $10^{4}$ точек и занимает $1 \mathrm{~s}$. Этот пример показывает, что сканирование квадруполя с высоким разрешением не очень практично. Квадруполи высокого разрешения действительно важны для тандемных инструментов, где они выполняют функцию селекции родительских ионов, для последующей фрагментации, a анализ спектра выполняется быстро и точно другими типами масс-анализаторов. Современные квадруполи могут обеспечить разрешающую способность на уровне 5000. Улучшение разрешения даже в 2 раза без существенной потери трансмиссии будет весьма значительным усовершенствованием технологии.

Нет сомнений, что новый режим работы имеет положительные последствия для общей технологии квадруполей. Можно будет изготавливать более короткие квадруполи (длиной $100 \mathrm{~mm}$ вместо $200 \mathrm{~mm}$ ) или расширять массовый диапазон за счет использования более низкой частоты RF, или использовать пучки ионов более высокой энергии. Приложения для космоса и миниатюрные квадруполи также смогут использовать преимущества нового режима работы, основанного на X-band.

Настоящая работа показывает теоретическую возможность улучшения характеристик квадрупольных фильтров масс. Авторы предвидят возможные сложности практической реализации. Фактическая разрешающая способность может быть просто ограничена стабильностью современных источников напряжения или температурной стабильностью инструмента. Поскольку предлагаемый метод не предполагает каких-либо модификаций механических частей квадруполей, а только изменение способа питания, представляется весьма возможным, что данный метод может быть успешно реализован на современном уровне технологии.

Работа выполнена при поддержке Российского фонда фундаментальных исследований (грант № 14-02-97505).

\section{Список литературы}

[1] Судаков М.Ю., Мамонтов Е.В. // ЖТФ. 2016. Т. 86. Вып. 11. C. 112-120.

[2] Paul W., Reinghard H.P., Von Zahn U. // Z. Phys. 1958. Vol. 152. P. 143-153.

[3] Du Z., Douglas D.J., Konenkov N.V. // J. Anal. At. Spectrom. 1999. Vol. 14. P. $1111-1119$.

[4] Sarfaraz U., Syed A.H., Hogan T., Gibson J., Taylor S. // J. Am. Soc. Mass Spectrom. 2012. Vol. 23. P. 988-995.

[5] Von Zahn U. // Z. Phys. 1962. Vol. 168. P. 129-142.

[6] Amad M.H., Houk R.S. // Anal. Chem. 1998. Vol. 70. P. 4885-4889.
[7] Konenkov N.V., Kratenko V.I. // Int. J. Mass Spectrom. Ion Processes. 1991. Vol. 108. P. 115-136.

[8] Ying J.-F., Douglas D.J. // Rapid Comm. Mass Spectrom. 1996. Vol. 10. P. 649-652.

[9] Du Z., Olney T.N., Douglas D.J. // J. Am. Soc. Mass Spectrom. 1997. Vol. 8. P. 1230-1236.

[10] Chen W., Collings B.A., Douglas D.J. // Anal. Chem. 2000. Vol. 72. P. 540-545.

[11] Titov V.V. // Int. J. Mass Spectrom. Ion Processes. 1995. Vol. 141. P. 45-56.

[12] Miseki K. // J. Mass Spectrom. Soc. Jpn. 2009. Vol. 51. P. 23-29.

[13] Konenkov N.V., Cousins L.M., Baranov V.I., Sudakov M.Yu. // Int. J. Mass Spectrom. 2001. Vol. 208. P. 17-27.

[14] Press W.H., Teukolsky S.A., Vetterling W.T., Flannery B.P. Numerical Recipes: The Art of Scientific Computing, Third Edition, NY: Cambridge University Press, 2007. 1235 p.

[15] Douglas D.J., Konenkov N.V. // Rapid Comm. Mass Spectrom. 2002. Vol. 16. P. $1425-1431$.

[16] Дуглас Д.Джс., Глебова Т.А., Коненков Н.В., Судаков М.Ю. // ЖТФ. 1999. Т. 69. Вып. 10. С. 96-101.

[17] Dahl D.A. SIMION 3D v.70 Users Manual. Idaho: National Eng. Envir. Lab., 2000. 366 p.

[18] Dawson P.H. Quadrupole Mass Spectrometry and its Applications. NY: American Institute of Physics, Woodbury, 1995. $343 \mathrm{p}$.

[19] Brubaker W.M. // US Patent 3.410.997. 1968.

[20] Zhao X., Xiao Z., Douglas D.J. // Anal. Chem. 2009. Vol. 81. P. 5806-5811. 